
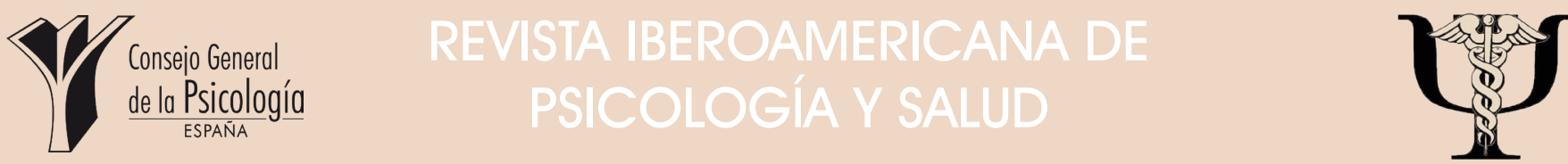

Revista Oficial de la Federación lberoamericana de Asociaciones de Psicología (FIAP) [Official Journal of the Latin-American Federation of Psychological Associations]

\title{
Dependencia emocional y estilo de apego adulto en las relaciones de noviazgo en jóvenes españoles
}

\author{
Laura Valle y María de la Villa Moral* \\ Universidad de Oviedo
}

- Recibido: 21 - 07 - 2017 - Aceptado: 28 - 11 - 2017 . Avance online: 18 - 12 - 2017

\begin{abstract}
RESUMEN: Un concepto ligado al de Dependencia Emocional es el de apego, de modo que en su propia definición se incluyen términos como apego patológico o invalidante e incluso se ha constatado que antecedentes personales de carencias afectivas infantiles y el apego adulto ansioso representan indicadores de semejante trastorno. Se propone como objetivo de este estudio analizar la relación entre la dependencia emocional y los diferentes tipos de apego adulto, así como su prevalencia y los perfiles en función del género, la edad y el historial de parejas. Para ello, han participado 382 adolescentes y jóvenes españoles con edades comprendidas entre los 18 y 35 años $(M=24,5 ; D T=1,978)$. Se ha determinado que el 23,3\% de los participantes muestran signos de dependencia emocional, siendo los varones los que puntúan más alto, así como los jóvenes que han tenido más de dos relaciones de noviazgo. Se ha demostrado la relación entre dependencia emocional y estilos de apego adulto, en el sentido de que el estilo seguro se asocia con la ausencia de DE y el estilo huidizo-temeroso, con los mayores niveles. Se aporta evidencia de la relación entre dependencia emocional y apego adulto y se discuten las implicaciones en las relaciones de pareja.

PALABRAS CLAVE: Dependencia Emocional, Estilos de apego adulto, Relaciones interpersonales, Jóvenes, Relaciones de pareja.
\end{abstract}

\section{Emotional dependence and adult attachment style in dating relationships in spanish young people}

ABSTRACT: A concept linked to Emotional Dependency is that of attachment, so that in its own definition are included terms such as pathological or invalidating attachment and it has even been found that personal history of childhood affective deficits and anxious adult attachment represent indicators of such disorder. Thus, the objective of this study is to analyze the relationship between emotional dependence and the different types of adult attachment, as well as their prevalence and profiles according to gender, age and the history of couples. To this end, 382 Spanish adolescents and young people aged 18-35 participated $(M=24.5$; DT $=1,978)$. It has been determined that $23.3 \%$ of the participants show signs of emotional dependence, with men being the ones who score the highest, as well as young people with whom they have had more than two dating relationships. The relationship between emotional dependence and adult attachment styles has been demonstrated, in the sense that safe style is associated with the absence of ED and the elusive-fearful style with the highest levels. Evidence is provided of the relationship between emotional dependence and adult attachment, and the implications of couple relationships are discussed.

KEYWORDS: Emotional Dependence, Adult attachment styles, Relationships, Young people, Couple relationships.

Las Dependencias Sentimentales o Afectivas (DS) representan un comportamiento desadaptativo contingente a una interrelación afectivodependiente (Sirvent, 2004) y se caracterizan tanto por elementos típicamente adictofílicos, tales como el vacío emocional, los

*Correspondencia: María de la Villa Moral Jiménez.

Universidad de Oviedo.

Código Postal: 33003, Facultad de Psicología, Plaza Feijóo, s/n

Despacho 211

E-mail:mvilla@uniovi.es

(C) 2018 Sociedad Universitaria de Investigación en Psicología y Salud. Publicado por Consejo General de Colegios Oficiales de Psicólogos, España. Este es un artículo Open Access bajo la CC BY-NC-ND licencia (http://creativecommons.org/licencias/by-nc-nd/4.0/)

Citar como/Cite as: Valle, L., y Moral, M. V. (2018). Dependencia emocional y estilo de apego adulto en las relaciones de noviazgo en jóvenes españoles. Revista lberoamericana de Psicología y Salud, 9(1), 27-41. https://doi.org/10.23923/i. rips.2018.01.013 síntomas de abstinencia o el craving, como por componentes vinculares y cognitivo-afectivos. Así pues, afectan a las relaciones de pareja y a la propia persona, siendo rasgos comunes en estos trastornos la necesidad ávida del otro, un enganche emocional y una preocupación excesiva por agradar a la persona de la que se depende, así como la manifestación de sentimientos negativos (culpa, abandono, inescapabilidad emocional, etc.). Otros indicadores son el autoengaño, el desvalimiento emocional, un estado de ánimo disfórico, el 
fantaseo y la euforia excesiva en lo referente a su relación de pareja, en la cual suelen adoptar un rol subordinado, asociado a su vez a una progresiva anulación personal (Moral y Sirvent, 2008, 2009).

Específicamente, la Dependencia Emocional (DE) es un tipo de dependencia sentimental que se da entre dos sujetos no adictos y que se describe como un patrón crónico de demandas afectivas frustradas sobre una persona que intentan satisfacerse mediante relaciones interpersonales de apego patológico (Sirvent y Moral, 2007). El término Dependencia Emocional se utiliza básicamente en la literatura de autoayuda, siendo escaso en la literatura científica (Sophia, Tavares y Zilberman, 2007). Así pues, resulta complejo identificar sus principales manifestaciones patognomónicas, dado que algunas de las conductas y estilos de pensamiento propios pasan inadvertidos por la dificultad del individuo para reconocer su problemática, así como por su aceptación social (Cogswell, Alloy, Karpinski y Grant, 2010).

Como características distintivas de los dependientes emocionales destacan su posesividad en las relaciones, en las que sufren un intenso desgaste afectivo, su incapacidad de romper ataduras y una voracidad de amor y cariño (Sirvent y Moral, 2007). En el caso del colectivo juvenil, la prolongación artificial de la adolescencia en sociedades en crisis ha dado lugar a un descenso de la competencia emocional en las últimas décadas, experimentando los jóvenes actuales cada vez más problemas emocionales, de ansiedad y de depresión (Moral y Ovejero, 2004), de ahí la pertinencia de centrar el presente estudio de DE en población joven. A nivel práctico, cada vez más se propone el estudio de las DE en jóvenes, como el establecimiento por parte de Lemos y colaboradores (2012) de un perfil cognitivo. Hoy en día, se estudia la influencia y/o relación de las dependencias sentimentales en diversos ámbitos, como la violencia de pareja (Aiquipa, 2015; Moral, García, Cuetos y Sirvent, 2007; Petruccelli et al., 2014) y las actitudes sexistas y la resolución de conflictos (Bringas et al., 2017; Pradas y Perles, 2012) o la ciberconducta (Espinar, Zych y Rodríguez-Hidalgo, 2015), entre otros. De este modo, en la última década, el estudio de la DE se ha incrementado y diversificado con numerosos estudios en los que se aborda esta problemática (véase Barraca, 2015; Izquierdo y Acosta, 2013; Lemos, Londoño y Zapata, 2007; Rodríguez-Medina, 2013; Santamaría et al., 2015). Por otro lado, se constata que en el amplio espectro de las relaciones de pareja, se manifiestan conductas violentas en mayor o menor medida (Batiza, 2017; Cala, Trigo y Saavedra, 2016; Medrano, Miranda y Figueras, 2017; Redding, Ruiz, Fernández y Guijarro, 2017). Esto se vincula con estudios en los que se resaltan los aspectos antisociales de la datación de actos juveniles violentos enraizados en conductas antisociales agresivas, sin señales de características específicas del delincuente, por lo que se cuestiona la validez de los programas de tratamiento específico del delito en prisión para jóvenes delincuentes de violencia en el noviazgo, de acuerdo con Siödin, Wallinius, Billstedt, Hofvander y Nilsson (2017).

Un concepto fuertemente ligado a las dependencias sentimentales es el del apego. Se valora que la diferencia entre el apego ansioso y la dependencia emocional se basa en el enfoque excesivamente conductual del primero, así como que aunque los dependientes emocionales presenten apego invalidante, lo contrario no es necesariamente cierto. A su vez, en la propia definición de las dependencias sentimentales (DS) se incluyen términos como apego patológico o invalidante, tomándose así los antecedentes personales de carencias afectivas infantiles y el apego ansioso como indicadores de este trastorno (Moral y Sirvent, 2009). De hecho, Sangrador (1993), justifica la aparición de este tipo de dependencia aludiendo a la inmadurez afectiva del individuo e incluso el propio Bowlby (1979) ya hacía referencia a la ansiedad de separación, típica de la DS, y a su similitud con el apego inseguro en aspectos tales como el temor a la pérdida de la figura vinculada, la búsqueda de proximidad y la protesta por la separación (Joel, McDonald y Shimotomai, 2010). En este sentido, Izquierdo y Acosta (2013) incluso se cuestionan acerca de si el apego es el origen de la dependencia afectiva, por lo que una historia de apego seguro podría actuar como factor de protección frente a conflictos en las relaciones afectivas futuras (Smith, Ortiz y Apodaca, 2014), 
tales como la DE, por lo que se considera de interés realizar un estudio que relacione ambos conceptos.

Desde la Teoría del Apego se abordan las relaciones afectivas según el grado de seguridad con el que los individuos exploran y se adaptan a su entorno. Dicha seguridad se enmarca en las relaciones significativas que se desarrollan a lo largo del ciclo vital en un ambiente de confianza y que se caracterizan por la comprensión y la receptividad (Ainsworth, Blehar, Waters y Wall, 1978). En este sentido, con el término apego adulto se hace referencia a los vínculos tempranos de protección en el desarrollo psicológico del individuo, de modo que el vínculo de apego se refiere al lazo afectivo en el que la persona vinculada percibe al otro como base de seguridad (Cassidy, 2008). Se trata de un vínculo afectivo estable y no transitorio, manteniéndose una relación con esa figura emocionalmente significativa y experimentándose angustia ante la separación de la misma, así como representa la búsqueda de seguridad en la relación, como principales características de semejante vínculo (Ainsworth, 1989; Cassidy, 2008). Así, las expectativas de apego afectarán al individuo en su grado de comodidad ante las relaciones íntimas, en sus habilidades de confianza, en su nivel de dependencia y en el temor al abandono (Simpson y Rholes, 1998). Por tanto, el establecimiento de un tipo de apego seguro en la infancia permitirá al sujeto autoconcebirse como un individuo valioso y digno de afecto, pudiendo así ejercer un rol positivo en las relaciones con los otros en etapas posteriores (Bowlby, 1979). De este modo, resulta de interés constatar que la interacción entre la edad y el nivel de instrucción materno explica en parte el nivel de base segura de las madres (Nóblega, Traverso, Ugarte y Caballero, 2017). A su vez, las interacciones con las primeras figuras de apego determinarán el estilo de apego adulto, mediante la organización de un conjunto de conductas, emociones, sentimientos y respuestas al ambiente que enmarcarán sus relaciones con los otros (Gillath et al., 2006) y moldearán el comportamiento adulto en las relaciones de pareja (Hazan y Shaver, 1987). Así pues, dado que las relaciones de pareja adultas implican vínculos afectivos y socioemocionales complejos, incluyendo los de cuidado y protección, pueden ser entendidas como procesos de apego (Hazan y Zeifman, 1999). En estudios como los de Guzmán y Trabucco (2014), se hallaron diferencias en la empatía diádica según los estilos de apego, de modo que las personas con estilos temerosos y desentendidos quienes mostraron preocupación empática.

Los pioneros en el estudio del apego adulto fueron, entre otros, Main, Kaplan y Cassidy (1985) y Hazan y Shaver (1987), quienes crearon una tipología análoga entre el apego infantil y el adulto, basándose en las tres estilos básicos de apego (seguro, ambivalente y evitativo) propuestos por Ainsworth y colaboradores (1978). Posteriormente, Bartholomew y Horowitz $(1990,1991)$ ampliaron estas categorías dada la evidencia en investigación de la existencia de cuatro estilos afectivo: seguro, preocupado, huidizo alejado/evitativo y huidizo temeroso. El estilo afectivo seguro se caracteriza por tener un modelo mental positivo y confianza tanto de/en sí mismo como de/en los demás, una elevada autoestima, ausencia de problemas interpersonales serios y deseo de intimidad, en la que se siente cómodo (Bartholomew y Horowitz, 1991; Mikulincer y Horesh, 1999). Estos individuos mantienen además el equilibrio entre sus necesidades afectivas y la autonomía personal (Mayseless, 1996), lo que conlleva a menudo a relaciones personales satisfactorias. Por el contrario, el individuo con estilo huidizo temeroso posee un modelo mental negativo tanto de sí mismo como de los demás y una baja confianza en ambos casos, necesitan intensamente la aprobación de los demás, consideran las relaciones como algo secundario a cuestiones profesionales y se sienten incómodos en situaciones íntimas (Bartholomew y Horowitz, 1991; Mayseless, 1996). Por su parte, en el estilo huidizo-alejado el modelo que el individuo tiene de sí mismo es positivo, pero es negativo hacia los demás (Bartholomew y Horowitz, 1991). Presentan estos sujetos una elevada autosuficiencia emocional, una baja activación de las necesidades de apego, considerando las relaciones como algo secundario a cuestiones materiales y se muestran altamente incómodos ante la intimidad (Mayseless, 1996). Por último, los sujetos con un estilo afectivo preocupado 
tienen un modelo mental negativo de sí mismo pero positivo de los demás (Bartholomew y Horowitz, 1991). Su autoestima es baja, presentan una elevada activación del sistema de apego y conductas de dependencia, mostrando una necesidad constante de aprobación y una preocupación excesiva por las relaciones. En cualquier caso, cabe destacar que se debe tener siempre en cuenta que los estilos de apego no han de ser empleados como categorías discretas, sino como continuas (Waters y Beauchaine, 2003).

En la actualidad, podemos encontrar diversos análisis acerca del impacto que el apego ejerce en las relaciones de pareja tanto de jóvenes como de adultos (véase Jensen, Willoughby, Holman, Busby y Shafer, 2015; Penagos, Rodríguez, Carrillo y Castro, 2006; Petani, 2011). Investigaciones recientes han arrojado datos que parecen confirmar la evidencia del efecto mediador del apego en la calidad de las relaciones de pareja (Martínez, Fuertes, Orgaz, Vicario y González, 2014; Smith, Ortiz y Apodaca, 2014), así como en la violencia de pareja (Loinaz y Echeburúa, 2012; Sánchez, 2016).

\section{- OBJETIVOS E HIPÓTESIS}

El principal objetivo del presente estudio es contribuir al establecimiento de un análisis diferencial entre los conceptos de dependencia emocional y apego adulto, así como relacionar ambos conceptos.

Como segundo objetivo de la investigación se plantea comprobar la existencia de diferencias significativas en los niveles de DE según el género, niveles de edad, el tener o no novio/a y el historial de parejas; determinando además la tasa de prevalencia de dicho trastorno entre los distintos grupos y en su totalidad. Asimismo, se hallará el tipo de apego adulto que los participantes presentan para, posteriormente, determinar si el estilo de apego correlaciona de forma estadísticamente significativa con las variables sociodemográficas propuestas, prestando especial atención a los niveles de DE.

Se plantean las siguientes hipótesis de investigación:
Se prevé hallar una correlación estadísticamente significativa entre los distintos estilos de apego y la existencia o no de DE, en el sentido de hallar una correlación positiva entre la presencia de DE y un tipo de apego inseguro.

Se hallarán diferencias significativas en los jóvenes con DE en función del género y la edad, siendo las mujeres más jóvenes quienes presenten mayor prevalencia. En cuanto al historial de parejas, se prevé que se hallarán diferencias significativas, de modo que los participantes con mayor número de parejas presentarán mayor prevalencia de DE.

En lo relativo al apego, se espera encontrar diferencias significativas según el género y la edad, siendo los varones de más edad quienes manifiesten principalmente un estilo de apego seguro.

Por último, se prevé que aquellos jóvenes con acusada DE muestren un estilo de apego preocupado, a quienes presenten niveles de DE leve les corresponderán estilos de apego huidizo-temeroso y huidizo-alejado, y el apego seguro será más identificativo de los jóvenes que apenas presenten signos de DE.

\section{MÉTODO}

\section{- PARTICIPANTES}

La muestra del presente estudio está compuesta por 382 participantes residentes en España, de los cuales 210 son mujeres (55\%) y 172 hombres (45\%) que han sido elegidos mediante un muestreo de tipo opinático. La edad de los encuestados fue previamente delimitada entre los 18 y 35 años debido a nuestro objeto de estudio. Siguiendo criterios psicosociales, se establecen tres grupos de edad: personas de entre 18 y 21 años $(26,2 \%)$, de entre 22 y 25 años $(42,1 \%)$ y mayores de 25 años $(31,7 \%)$.

En cuanto al lugar de procedencia, encontramos el mayor porcentaje de participación en Galicia (27,2\%), seguidamente de Andalucía (1 1,3\%), Asturias (9,4\%), Madrid $(8,1 \%)$, Cataluña $(7,9 \%)$, Comunidad Valenciana (5,2\%), Castilla y León (4,7\%), Cantabria (3, 1\%), Aragón y Región de Murcia (2,4\% ambos), Castilla-La Mancha (2,1\%), País Vasco e Islas 
Canarias (1,8\% ambos), Extremadura (1,3\%), Navarra (0,8\%) y La Rioja (0,3\%). Sumando estos a aquellos encuestados que simplemente contestaron "España" nos encontramos con un total del 98,2\% de personas nacidas en este país, frente al 1,8\% de otras nacionalidades.

Con respecto al nivel formativo, un 3,4\% de los participantes finalizaron los estudios básicos (ESO), un 27,2\% los estudios medios (Bachillerato y/o Ciclos Formativos) y la mayoría $(69,4 \%)$ cuentan con estudios superiores (Universitarios).

Ante la pregunta de si tienen pareja actualmente, un $50,5 \%$ de los jóvenes respondieron afirmativamente, y un $49,5 \%$ en sentido contrario. Respecto al número de parejas que los participantes afirman haber tenido (contando la actual en caso de tenerla) comprende un rango de 0 a 17. Tras calcular la tendencia central (media 2,38; mediana y moda, $2)$, se establecieron tres grupos: aquellos que han tenido menos de dos parejas $(32,7 \%)$, los que han respondido "dos" (28,3\%) y aquellos que han tenido más de dos relaciones (39\%).

\section{- INSTRUMENTOS DE MEDIDA}

En el presente estudio se midieron las variables sociodemográficas género, edad, lugar de procedencia, nivel formativo, el tener o no pareja en la actualidad y el historial de parejas de los participantes, y las variables Dependencia Emocional y Apego Adulto.

Para medir la presencia o ausencia de Dependencia Emocional así como sus niveles se utilizó el Inventario de Relaciones Interpersonales y Dependencias Sentimentales (IRIDS-100; Sirvent y Moral, 2005), anterior TDS-100. Este cuestionario valora las Dependencias Relacionales y cuenta con tres subescalas de Dependencia Sentimental (DE, Codependencia y Bidependencia) y según la estructura factorial obtenida por rotación varimax está integrado por siete factores de relación interpersonal (Necesidad del otro, Búsqueda de sensaciones, Acomodación, Autoengaño, Sentimientos adversos, Identidad/Caracteriosis y Antecedentes personales). Aunque en su versión original consta de 100 ítems, en este estudio se han utilizado 81 de ellos elegidos por acuerdo interjueces dado que nuestro estudio se enfoca en la subescala de Dependencia Emocional y en los factores pertinentes al objeto de estudio. La escala de respuesta es de tipo Likert comprendiendo valores de 1 (muy en desacuerdo) a 5 (muy de acuerdo). En el análisis de fiabilidad de esos 81 ítems seleccionados se ha obtenido un alfa de Cronbach de 0,957, un índice muy alto.

Para medir los distintos estilos de apego se usó el Cuestionario de Apego Adulto (Melero y Cantero, 2008), con base en el Cuestionario de Relación (CR, Bartholomew y Horowitz, 1991), que evalúa los estilos de apego adulto en población no clínica y está validado en población española. Según el análisis factorial, el cuestionario se compone de 40 ítems, con una escala de respuesta tipo Likert de 6 puntos, siendo 1 nada de acuerdo y 6 muy de acuerdo, y nos proporciona 4 factores que explican el $40 \%$ de la varianza total (Baja autoestima, necesidad de aprobación y miedo al rechazo; Resolución hostil de conflictos, rencor y posesividad; Expresión de sentimientos y comodidad con las relaciones y Autosuficiencia emocional e incomodidad con la intimidad). Este instrumento permite clasificar a los sujetos en Apego seguro e inseguro, y además discrimina entre los tres tipos de inseguridad: alejado, preocupado y temeroso-hostil. En nuestra muestra se obtiene una fiabilidad total medida mediante el indicador alfa de Cronbach de 0,879 .

\section{- PROCEDIMIENTO Y ANÁLISIS DE DATOS}

Para la recopilación de datos, los instrumentos utilizados se aplicaron bajo el modelo de encuesta auto aplicada vía online que se mantuvo activa durante los meses de enero a marzo de 2017, firmando los participantes un acuerdo de consentimiento. Se ha garantizado el anonimato de los participantes y se aportó una declaración de responsabilidad sobre el empleo de las respuestas aportadas para fines exclusivos de investigación.

Respecto a los análisis de datos, en primer lugar, se han empleado análisis descriptivos de las variables DE y Apego Adulto mediante estadísticos de tendencia central y distribución. A continuación se empleó un Análisis de Conglomerados con método de participación k-medias para poder clasificar a los participantes en los distintos tipos y estilos de apego. El estudio 
de las diferencias en la variable dependiente Dependencia Emocional en función de las variables género y tener pareja se llevó a cabo a través de la prueba $T$ de comparaciones de medias para muestras independientes, dado que estas variables sólo cuentan con dos niveles; mientras que en función de la edad y el historial de parejas se llevó a cabo empleando un ANOVA unifactorial de efectos intersujeto, dado que estas variables cuentan con tres niveles de respuesta. Por último, se emplearon técnicas correlacionales para analizar la relación entre el Apego Adulto y el resto de variables. El estadístico empleado para tal fin fue Chi2 de Pearson.

El análisis de los datos fue realizado en su totalidad con el software informático SPSS versión 21.

\section{RESULTADOS}

En primer lugar, se exponen las tasas de prevalencia de dependencia emocional en los participantes del estudio (véase Tabla 1). Cabe resaltar que en los estudios realizados por Sirvent y Moral (2008) Ilevados a cabo en población general, la tasa de prevalencia de DE era del $11 \%$; mientras que en nuestra muestra con jóvenes esta cifra asciende al 23,3\%. Asimismo, se ha hallado que la mayoría de personas que presentan dependencia emocional, lo hacen en un grado intenso, de modo que un $10,2 \%$ de los participantes muestran una DE intensa, el 6\% DE acusada y el $7,1 \%$ DE leve (véase Tabla 1).

\begin{tabular}{|c|c|c|}
\hline \multicolumn{3}{|c|}{$\begin{array}{c}\text { Tabla } 7 \\
\text { Tasas de prevalencia de DE }\end{array}$} \\
\hline & Frecuencia & Porcentaje válido \\
\hline Ausencia DE & 293 & $76,7 \%$ \\
\hline Presencia DE & 89 & $23,3 \%$ \\
\hline DE Leve & 27 & $7,1 \%$ \\
\hline DE Acusada & 23 & $6 \%$ \\
\hline DE Intensa & 39 & $10,2 \%$ \\
\hline
\end{tabular}

A su vez, para poder clasificar a los sujetos, primero fue necesario analizar los estadísticos de tendencia central y de distribución de las cuatro subescalas del CAA (Tabla 2). Los percentiles se han formado siguiendo el criterio teórico de Melero y Cantero (2005) para siete grupos iguales con la finalidad de poder clasificar las puntuaciones entre un valor "muy bajo" a uno "muy alto".

A continuación, se realizó una clasificación de los participantes en función de su estilo de apego, para ello fue necesario emplear dos análisis de conglomerados por el método de partición k-medias: uno que nos divida a los sujetos en Apego seguro o inseguro y otro que los clasifique en función de los cuatro estilos de apego adulto previamente descritos.

Estadísticos descriptivos de Apego Adulto

\begin{tabular}{|c|c|c|c|c|c|}
\hline & Escala 1 & Escala 2 & Escala 3 & Escala 4 & \\
\hline Percentiles & & & & & Valor \\
\hline $87-99$ & $54-64$ & $39-52$ & $40-44$ & $28-36$ & Muy alto \\
\hline $72-86$ & $49-53$ & $34-38$ & $38-39$ & $25-27$ & Alto \\
\hline $58-71$ & $44-48$ & $31-33$ & $36-37$ & $22-24$ & Mod./ Alto \\
\hline $44-57$ & $40-4$ & $28-30$ & $34-35$ & 21 & Moderado \\
\hline $30-43$ & $36-39$ & $25-27$ & 33 & $19-20$ & Mod./ Bajo \\
\hline $15-29$ & $31-35$ & $22-24$ & $29-32$ & $17-18$ & Bajo \\
\hline $1-14$ & $20-30$ & $13-21$ & $22-28$ & $12-16$ & Muy Bajo \\
\hline Media & 41,41 & 29,27 & 33,92 & 21,14 & \\
\hline DT & 10,24 & 8,31 & 5,03 & 5,37 & \\
\hline Mínimo & 18 & 11 & 20 & 10 & \\
\hline Máximo & 65 & 57 & 45 & 39 & \\
\hline
\end{tabular}


En referencia a la clasificación en dos grupos, el primer clúster, correspondiente al Apego seguro $(N=197)$ presenta una puntuación baja (34) en las escalas 1 (Baja autoestima, necesidad de aprobación y miedo al rechazo) y 2 (24; Resolución hostil de conflicto, rencor y posesividad). En la escala 3 (Expresión de sentimientos y comodidad en las relaciones) su puntuación es moderada (34) y en la escala 4 (Autosuficiencia emocional e incomodidad con la intimidad) es moderadamente baja (20). El segundo clúster, correspondiente al Apego inseguro $(N=185)$ tiene una puntuación alta en las dos primeras escalas $(50$ y 34 respectivamente), una puntuación moderada en la tercera escala $(34$, al igual que el primer clúster) y una puntuación moderadamente alta (23) en la escala 4. Las diferencias entre los correspondientes clústeres son significativas ( $p<$ 0.05) en las escalas 1, 2 y 4, aquellos factores que evalúan aspectos teóricamente relacionados con la inseguridad afectiva; mientras que en el tercer factor, relacionado con la seguridad, no se hallaron diferencias significativas entre conglomerados (Tabla 3). Así pues, un 51,57\% de los sujetos encuestados se clasifican dentro de un estilo de Apego seguro, y el 48,43\% restante, en un estilo de Apego inseguro.
En cuanto a la clasificación de los participantes en cuatro clústers en función de los cuatro estilos de apego teorizados (Figura 1 y Tabla 4), obtenemos que en el clúster 1, correspondiente al estilo de Apego huidizo-alejado $(N=72)$, se puntúa alto en la escala 2 (Resolución hostil de conflictos, rencor y posesividad), moderadamente alto en la escala 4, moderadamente bajo en la escala 1 y bajo en la escala relacionada con la seguridad. En el clúster 2, correspondiente al Apego seguro $(N=128)$, se puntúa bajo en las dos primeras escalas, moderadamente bajo en la escala 4 y moderadamente en la escala de seguridad. En el clúster 3, correspondiente a un estilo de Apego preocupado $(N=102)$, se puntúa moderadamente alto en la escala 1 (Baja autoestima, necesidad de aprobación y miedo al rechazo), de forma moderadamente baja en la escala 2 y tiene puntuaciones bajas en las escalas 3 y 4 . El clúster 4, correspondiente al Apego huidizo-temeroso $(N=80)$ presenta elevadas puntuaciones en las escalas relacionadas con la inseguridad afectiva, y moderadas en el tercer factor (expresión de sentimientos y comodidad con las relaciones.

Asimismo, tras aplicar un ANOVA, encontramos diferencias significativas entre todos los grupos en todas las dimensiones

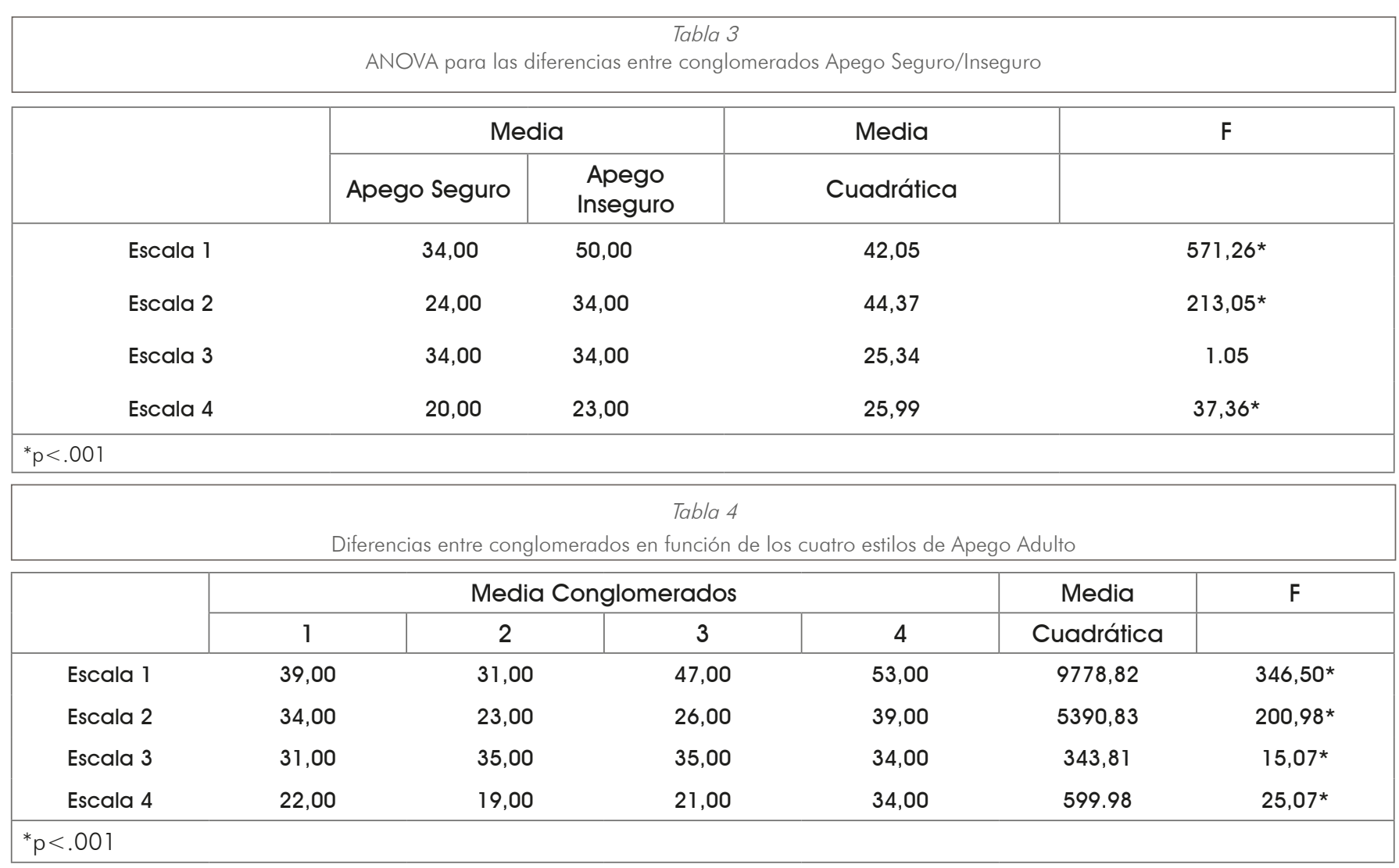


analizadas en función de los cuatro tipos de apego adulto (Tabla 4). Los porcentajes de clasificación, por tanto, son los siguientes: estilo seguro, 33,5\%; estilo preocupado, 26,7\%; estilo huidizo-temeroso: $20,9 \%$, y estilo huidizoalejado: 18,8\%.

Para comprobar si existe relación entre los estilos de apego adulto y la presencia o ausencia de Dependencia Emocional se ha empleado el estadístico de correlación de Pearson. Se ha comprobado que existe una relación estadísticamente significativa entre ambas variables $\left(\chi_{3}^{2}=60,765, p<0,001\right)$. Las mayores diferencias se dan entre los estilos de apego seguro e huidizo-temeroso. Las personas con estilo seguro son aquellas que con mayor frecuencia no presentan signos de DE; mientras que las que más manifiestan presencia de $\mathrm{DE}$ son aquellas con un estilo huidizo-temeroso (Figura 1). La fuerza de asociación es moderada $(C=0,37 ; p<0,01)$. También se ha aplicado el coeficiente de correlación de Spearman ( $p=$ ,238) que nos indica una baja correlación.

Respecto al análisis diferencial propuesto en DE en función del género, la edad, así como el historial de parejas, se ha comprobado que existen diferencias significativas $(t=2,100 ; p<$ ,05), obteniendo los hombres una puntuación media más alta en dependencia emocional.

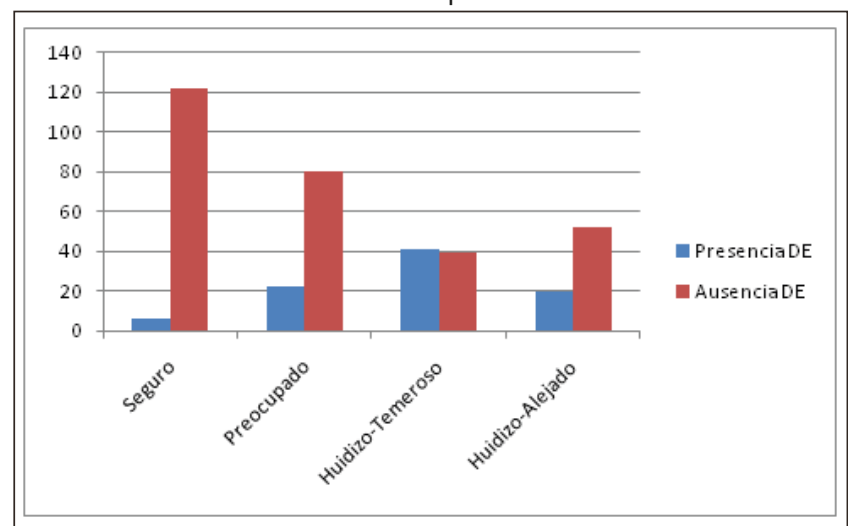

Figura 1. Casos de DE según el Estilo de Apego
A su vez, se ha comprobado que no existen diferencias significativas entre los grupos de edad en función de los niveles de DE $(F=1.518, p=.220)$. Por el contrario, al relacionar el historial de parejas y los niveles de DE se ha confirmado que existen diferencias significativas entre al menos dos de los niveles de la variable Número de parejas $\left(F_{2,379}=\right.$ $6,132, p=, 002)$ si bien el tamaño del efecto es pequeño (eta ${ }^{2}$ parcial $=, 031$ ). Mediante el contraste de Scheffé se ha comprobado que las diferencias se concentran entre los niveles "Menos de dos parejas" y "Más de dos parejas" $(p=0,002)$, de manera que las personas que han tenido más de dos parejas obtuvieron una puntuación media más alta en Dependencia Emocional (véase Tabla 5).

Para comprobar si existe relación entre los estilos de apego adulto y las variables demográficas género y edad se empleó el estadístico Chi-cuadrado de Pearson, de modo que en los casos que la asociación fue comprobada, se ha utilizado el coeficiente de contingencia y la prueba Tau-C de Kendall para comprobar si esa relación es o no lineal. Se comprueba que no existe relación entre los estilos de apego con las variables género, tener o no novio/a actualmente. Por el contrario, sí hay asociación entre apego adulto y edad (Tabla 6). Dado que la variable edad presenta una relación significativa con los estilos de apego $\left(\chi_{6}^{2}=22,93, p=, 001\right)$, se midió dicha asociación. Las mayores diferencias se dan en los estilos seguro y preocupado. Las personas de más de 25 años tienden a un Estilo preocupado, mientras que las de 22-25 años a uno seguro. La asociación entre edad y estilo de apego no es demasiado elevada $(C=, 238$, $p=, 001)$ y la relación no es de tipo lineal (Tau $\mathrm{C}$ de Kendall=-,059, $p=$,218).

\section{Tabla 5}

Diferencias en DE en función del $n^{\circ}$ de parejas

\begin{tabular}{|c|c|c|c|c|}
\hline Origen & Media Cuadrática & $\mathrm{F}$ & Sig. & Eta $^{2}$ parcial \\
\hline Modelo corregido & 2,119 & 6,12 & 002 & ,031 \\
\hline Intersección & 1459,168 & 4222,084 &, 000 & 918 \\
\hline Número de parejas & 2,119 & 6,132 &, 002 & 031 \\
\hline
\end{tabular}


Tabla 6

Resumen de los coeficientes Chi-cuadrado de Pearson para los Estilos de Apego

\begin{tabular}{|c|c|c|}
\hline Variable & Valor de $\chi^{2}$ & Sig. bilateral \\
\hline Género & 7,27 &, 064 \\
Edad & 22,93 &, 001 \\
Tener o no pareja & 2,424 &, 489 \\
No de parejas & 6,98 &, 323 \\
\hline
\end{tabular}

Finalmente, a objeto de determinar la relación entre los estilos de apego adulto y los niveles de Dependencia Emocional se ha empleado el estadístico de correlación de Pearson. Se ha comprobado que existe una relación estadísticamente significativa $\left(\chi^{2}{ }_{9}=69\right.$, 079, $p=, 001)$, de manera que las mayores diferencias se dan entre los estilos seguro y temeroso-huidizo (véase Figura 3). Los jóvenes con un estilo de apego seguro tienden a no presentar Dependencia Emocional, mientras que las personas con estilo temeroso-huidizo son las que mayores niveles presentan. La fuerza de asociación es moderada $(C=, 391, p \leq, 01)$ y la relación es linealmente positiva (Tau-C $=, 158, p$ $\leq, 01)$. Dado que las variables son cualitativasordinales también se ha aplicado el estadístico de correlación de Spearman, hallándose una relación positiva, aunque moderada, entre ambas variables $(p=, 350, p<, 01)$.

\section{DISCUSIÓN}

El principal propósito del presente estudio ha sido comprobar si existe una relación significativa entre los conceptos de Dependencia Emocional y Apego Adulto. En efecto, se obtuvo una correlación positiva tanto entre los estilos de apego y la existencia de DE, como con su intensidad. De esta forma, en el presente estudio sólo seis participantes $(4,69 \%)$ con un Apego Seguro manifestaron presencia de DE, lo que concuerda con nuestra primera hipótesis. Esto se relaciona con los hallazgos de Bornstein, Geiselman, Eisenhart y Languirand (2002) sobre los perfiles relacionales, afirmando que aquellos individuos con sobredependencia destructiva experimentan ansiedad ante la cercanía, intimidad y disponibilidad de sus cuidadores, sumada a una extrema preocupación ante el abandono; comportamientos que pueden ser

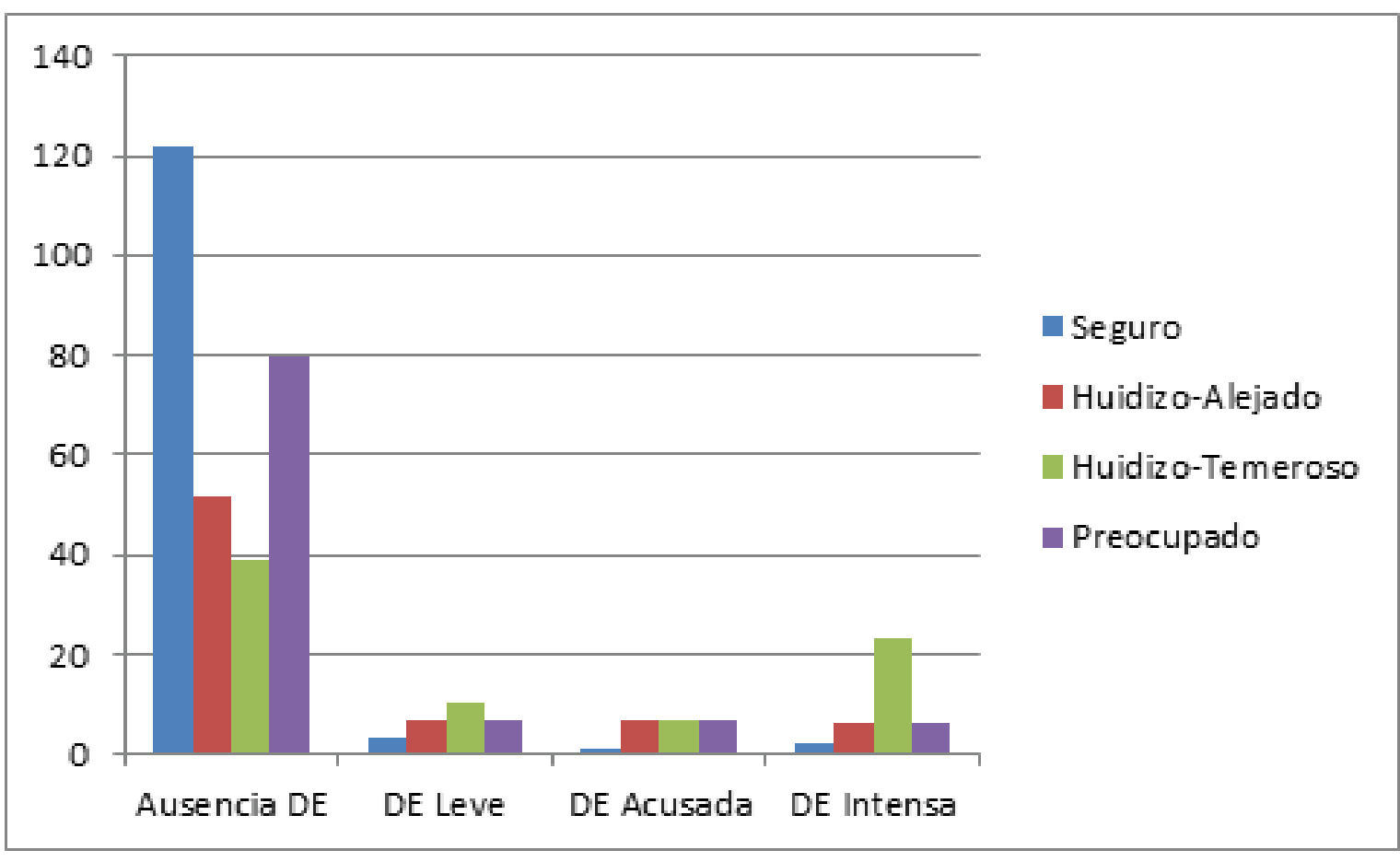

Figura 2. Relación entre Estilos de Apego Adulto y Niveles de Dependencia Emocional 
todos comprendidos desde el temor a la soledad, la ansiedad de separación y la búsqueda de expresión afectiva, tan característicos de la DE (Lemos y Londoño, 2006). Asimismo, en universitarios ecuatorianos Boada, Aguilera y Llerena (2015) demostraron la influencia recíproca entre la DE y los vínculos afectivos: la DE fomenta vínculos afectivos inseguros, y éstos a su vez, perpetúan las relaciones inestables de DE. Así, un tercio de los participantes en ese estudio presentaron conjuntamente DE y vínculos afectivos inseguros; mientas que la mayoría de estudiantes que no presentaron DE manifestaron vínculos seguros (69\%).

En cuanto a los niveles de Dependencia Emocional se han establecido tasas de prevalencia obteniendo como resultado que un $23,3 \%$ de los participantes presentan DE, de los cuales en su mayoría $(10,2 \%)$ manifiestan una DE intensa. No se han encontrado diferencias significativas en función de la edad, pero sí con relación al género y al historial de parejas. Contrariamente a lo hipotetizado en investigaciones anteriores, tales como la de Alonso-Arbiol, Shaver y Yárnoz (2002) o la de Ehrenberg y Saffrey (2007) que mostraban una tendencia más elevada en adolescentes de género femenino a establecer relaciones emocionalmente dependientes, han sido los hombres quienes han puntuado más alto en la escala de DE, así como aquellos participantes que han tenido más de dos parejas. Estas diferencias han sido expuestas previamente por autores como Moral y Sirvent (2009) quienes caracterizan a los varones con DE como buscadores de sensaciones fuertes e hiper-estimuladores; mientras que las mujeres dependientes tendrían como principal rasgo el no tomar conciencia real del problema debido a los procesos de autoengaño asociados a mecanismos de negación y no afrontamiento. Así, en otras investigaciones como la de Urbiola y Estévez (2015) con jóvenes españoles se comprobó que los chicos puntúan más alto en DE que las chicas, si bien también hallaron diferencias en función de la edad. En cambio, en estudios sobre la experiencia de victimización se constata que la tipo verbal emocional fue la más frecuente en chicas de menores edades (Redondo, Inglés y García, 2017), si bien en el estudio de Novo, Herbón y Amado (2016) no se encontró relación entre el género y la victimización de violencia de pareja con las tácticas de resolución de conflictos y el apego adulto.

En lo referente a la clasificación de los participantes en los cuatro estilos afectivos teorizados, no se han hallado diferencias en cuanto al género, ni en lo relativo al historial de parejas, pero sí en función de la edad. Han sido los jóvenes de entre 22 y 25 años las que han mostrado en su mayoría un estilo seguro y no los mayores de 25 años, quienes presentan mayormente un estilo preocupado. En este sentido, Melero y Cantero (2008) indicaban que empleando el CAA algunos sujetos seguros podrían confundirse bajo la etiqueta de huidizo-alejado debido a que su característica de autonomía puede conllevar a una elevada puntuación en autosuficiencia. Estos autores también justificaban su alto porcentaje de sujetos huidizos-alejados $(29,66 \%)$ atendiendo a los modelos actuales de socialización, en los que se sobrevalora la individualidad y la autosuficiencia frente al establecimiento de relaciones íntimas. En nuestro estudio, el porcentaje de participantes clasificados como huidizos-alejados se sitúa en un $18,84 \%$ siendo la categoría con menos sujetos. Cabe destacar que en la investigación realizada por Sánchez (2011) sí se hallaron diferencias en el estilo de apego en función del género y del número de parejas, obteniendo que los hombres tienden a poseer un estilo seguro, así como aquellas personas con un mayor número de parejas. Asimismo, CantúRodríguez (2016) también comprobaron una relación entre el tipo de apego y el fenómeno de las parejas intermitentes, afirmando que un miembro de una pareja intermitente es probable que posea un estilo de apego inseguro, pero que no todas las personas con apego inseguro formarán parte de una pareja intermitente ya que la interacción de ambos individuos juega un papel relevante. Asimismo, se han constatado asociaciones positivas entre la vinculación afectiva en la infancia y la calidad de la relación, comprobándose además el efecto mediador del apego actual (Martínez et al. 2014).

Por último, también se ha confirmado la existencia de relación entre los estilos de apego adulto y la intensidad de DE. Tal como habíamos 
teorizado, la mayoría de personas clasificadas dentro de un estilo seguro apenas han mostrado signos de DE, aunque por el contrario, han sido los clasificados en el estilo huidizo-temeroso y no en el preocupado los que han manifestado mayores niveles de dependencia. Otras investigaciones han mostrado que los sujetos seguros tienen una visión realista tanto de sí mismos como de los demás, lo que les permite regular los afectos sin una gran distorsión en sus representaciones mentales (Mikulincer y Horesh, 1999). Asimismo, Bartholomew (1990) afirmaba que los sujetos preocupados y temerosos comparten una dependencia hacia los demás, quizás explicada por su falta de identificación con los otros relacionada a su vez con la seguridad en las relaciones y la autovaloración (Yárnoz, Alonso-Arbiol, Plazaola y Sainz, 2001). Estos resultados pueden relacionarse con los hallazgos de Hurtado y Marchan (2016) acerca de la relación entre los estilos de apego y la violencia doméstica. Así pues, determinaron que un $77 \%$ de las mujeres víctimas de violencia doméstica presentan un estilo de apego ansioso (por su descripción, el equivalente al estilo preocupado: modelo mental negativo de sí mismo y positivo de los demás). Específicamente, este estilo ansioso o preocupado se relaciona con la violencia psicológica debido al miedo a la separación, la dependencia hacia el agresor, una constante necesidad de aprobación, una preocupación excesiva por las relaciones y la falta de objetividad de la mujer al evaluar los aspectos negativos de su relación, lo que se asemeja en gran medida a la caracterización de la Dependencia Emocional.

Como principales limitaciones de este estudio, hay que tener en cuenta que estos resultados deben ser considerados teniendo en cuenta el tamaño de la muestra, así como su parcial representatividad, al tratarse de participantes voluntarios y no tener la posibilidad de obtener unos grupos de edad balanceados. Otras limitaciones son las relativas al empleo de medidas autoadministradas, que aunque tienen ventajas como la reducción del tiempo en la recogida de datos, también pueden verse afectadas por la deseabilidad social, lo que puede influir directamente en las puntuaciones obtenidas (Novo, Fariña, Seijo y Arce, 2012).
Otra limitación es la relativa a la aplicación online del test, que puede dar lugar a la suplantación de identidad de los encuestados, aunque para minimizar este efecto se requería que los participantes iniciasen una sesión privada desde su cuenta de correo electrónico.

Para futuras investigaciones, es importante considerar recabar una muestra más amplia y representativa en la que el método de selección de los participantes sea probabilístico en aras de la extrapolación de los resultados. Sería interesante también poder estudiar estas variables en función de otras, como por ejemplo, el nivel formativo, el lugar de procedencia (especialmente comparando ámbitos rurales y urbanos); y ampliar este estudio a los menores de 18 años. Asimismo, consideramos de interés profundizar más acerca de posibles factores determinantes de la Dependencia Emocional y, específicamente, también resultaría interesante poder realizar un estudio longitudinal en el que se relacionasen el apego infantil, el adulto y la DE.

Con todo ello, podrían diseñarse programas de prevención e intervención que promuevan la optimización de nuestros vínculos socioafectivos, los patrones comunicacionales interpersonales, así como las competencias de inteligencia emocional intra e interpersonal con el fin de mejorar nuestros recursos relaciones y poder así prevenir la manifestación de apego patológico y/o intervenir con programas integrales de salud socioafectiva en población infantojuvenil.

\section{- Conflicto de intereses}

Los autores declaran no tener ningún conflicto de intereses.

\section{REFERENCIAS}

Ainsworth, M. D. S. (1989). Attachment beyond infancy. American Psychologist, 44(4), 709$716 . \quad$ http://dx.doi.org/10.1037/0003066X.44.4.709

Ainsworth, M., Blehar, M. Waters, E., \& Wall, S. (2015). Patterns of attachment: A psychological study of the strange situation. New York: Taylor \& Francis.

Aiquipa, J. J. (2015). Dependencia emocional en mujeres víctimas de violencia de pareja. Revista de Psicología, 33(2), 412-436. 
Alonso-Arbiol, I., Shaver, P., \& Yárnoz, S. (2002). Insecure attachment, gender roles and interpersonal dependency in the Basque Country. Personal Relationships, 9, 479490. http://dx.doi.org/ 10.1111/14756811.00030

Barraca, J. (2015). Una forma de dependencia emocional: El "Síndrome de Fortunata". Papeles del Psicólogo, 36(2), 145-152.

Bartholomew, K. (1990). Avoidance of intimacy: An attachment perspective. Journal of Social and Personal Relationships, 7, 147-178. http:// dx.doi.org/10.1177/0265407590072001

Bartholomew, K., \& Horowitz, L. (1991). Attachment styles among young adults: A test of a four category model. Journal of Social and Personal Psychology, 61, 226-244. http:// dx.doi.org/10.1037/0022-3514.61.2.226

Batiza, F. J. (2017). La violencia de pareja: Un enemigo silencioso. Archivos de Criminología, Seguridad Privada y Criminalística, 18, 144151.

Boada, B. Aguilera, R. P., \& Llerena, A. G. (2015). Dependencia emocional y vínculos afectivos en estudiantes de la carrera de Psicología Clínica de la Universidad Nacional de Chimborazo [Tesis de Fin de Grado]. Riobamba, Ecuador: Universidad Nacional de Chimborazo.

Bornstein, R. F., Geiselman, K. J., Eisenhart, E. A., \& Languirand, M. A. (2002). Construct validity of the Relationship Profile Test: Links with attachment, identity, relatedness, and affect. Assessment, 9(4), 373-381. http://dx.doi. org/10.1177/1073191102238195

Bowlby, J. (1979). The making \& breaking of affectional bonds. New York, NY: Routledge.

Bringas-Molleda, C., Estrada-Pineda, C., SuárezÁlvarez, J., Torres, A., Rodríguez-Díaz, F. J., García-Cueto, E., \& Rodríguez-Franco, L. (2017). Actitud sexista y trascendente durante el noviazgo entre universitarios latinoamericanos. Revista Iberoamericana de Psicología y Salud, 8(1). https://doi.org/10.23923/i. rips.2017.08.005

Cala, M. J., Trigo, E., \& Saavedra, F. J. (2016). Women's disengagement from legal proceedings for intimate partner violence sociodemographic and psychosocial variables.
European Journal of Psychology Applied to Legal Context, 8(1), 35-42. https://dx.doi. org/10.1016/i.ejpal.2015.10.002

Cantú-Rodríguez, M. B. (2016). Mujeres en parejas intermitentes y su percepción de la ansiedad en la relación. Revista Electrónica de Psicología de Iztacala, 19(2), 719-736.

Cassidy, J. (2008). The nature of child's ties. En J.Cassidy \& P. R. Shaver (Eds.), Handbook of attachment: Theory, research, and clinical implications (pp. 3-22). New York, NY: The Guilford Press.

Cogswell, A., Alloy, L., Karpinski, A., \& Grant, D. (2010). Assessing Dependency using selfreport and indirect measures: Examining the significance of discrepancies. Journal of Personality Assessment, 92(4), 306-316. http://dx.doi.org/10.1080/00223891.2010 .481986

Ehrenberg, M., \& Saffrey, C. (2007). When thinking hurts: Attachment, rumination and postrelationship adjustment. Personal Relationships, 14, 351-368. http://dx.doi. org/10.1111/i.1475-6811.2007.00160.x

Espinar, E., Zych, I., \& Rodríguez-Hidalgo, A. J. (2015). Ciberconducta y dependencia emocional en parejas jóvenes. Psychology, Society \& Education, 7(1), 41-55.

Gillath, O., Mikulincer, M., Fitzsimons, G., Shaver, P., Schachner, D., \& Bargh, J. (2006). Automatic activation of attachment related goals. Personality and Social Psychology Bulletin, 32(10), 1375-1388. http://dx.doi. org/10.1177/0146167206290339

Guzmán, M., \& Trabucco, C. (2014). Estilos de apego y empatía diádica en relaciones de pareja en adultos emergentes. Revista Argentina de Clínica Psicológica, 23(1), 61 70. Recuperado de http://www.redalyc.org/ articulo.oa? id =281935591006

Hazan, C., \& Shaver, P. (1987). Romantic love conceptualized as an attachment process. Journal of Personality and Social Psychology, 52, 511-524. http://dx.doi. org/10.1037/0022-3514.52.3.511

Hazan, C., \& Zeifman, D. (1999). Pair bonds as attachments: Evaluating the evidence. En J. Cassidy \& P. Shaver (Eds.), Handbook 
of attachment: Theory, research, and clinical applications (pp. 336-354). New York, NY: The Guildford Press.

Hurtado, E. T., \& Marchan, D.A. (2016). Estilos de apego y violencia doméstica en mujeres atendidas en la unidad judicial especializada en violencia contra las mujeres y la familia del consejo de la judicatura de Riobamba [Trabajo de Fin Grado]. Riobamba, Ecuador: Universidad Nacional de Chimborazo.

Izquierdo-Martínez, S. A., \& Gómez-Acosta, A. (2013). Dependencia afectiva: Abordaje desde una perspectiva contextual. Psychologia. Avances de la Disciplina, 7(1), 81-91.

Jensen, T., Willoughby, J., Holman, T., Busby, D., \& Shafer, K. (2015). Associations between family and Interpersonal processes and emerging adult marital paradigms: Does adult attachment mediate? Journal of Adult Development, 22(1), 50-62. http://dx.doi.org/10.1007/s10804014-9200-3

Joel, S., MacDonald, G., \& Shimotomai, A. (2010). Conflicting pressures on romantic relationships commitment for anxiously attached individuals. Journal of Personality, 79(1), 51-74. http://dx.doi.org/10.1111/ j.1467-6494.2010.00680.x

Lemos, M., \&Londoño, N. H. (2006). Construcción y validación del Cuestionario de Dependencia Emocional en población colombiana. Acta Colombiana de Psicología, 9(2), 127-140.

Lemos, M., Londoño, N., \& Zapata, J.A. (2007). Distorsiones cognitivas en personas con dependencia emocional. Informes Psicológicos, 9, 55-69.

Lemos, M., Jaller, C., González, A. M., Díaz, Z. T., \& De La Ossa, D. (2012). Perfil cognitivo de la dependencia emocional en estudiantes universitarios en Medellín, Colombia. Universitas Psychologica., 17 (2), 395-404.

Loinaz, I., \& Echeburúa, E. (2012). Apego adulto en agresores de pareja. Acción Psicológica, 9(1), 33-46.

Main, M., Kaplan, K., \& Cassidy, J. (1985). Security in infant childhood and adulthood: A move to the level of representation. En I. Bretherton \& E. Waters, Growing points of attachment. Monographs of the society for research in child development. Monographs of the Society for Research in child Development. 1-2. Vol. 50. 1985. pp. 147-166. Serial \#209

Martínez-Álvarez, J. L., Fuertes-Martín, A., OrgazBaz, B., Vicario-Molina, I., \& GonzálezOrtega, E. (2014). Vínculos afectivos en la infancia y calidad en las relaciones de pareja de jóvenes adultos: El efecto mediador del apego actual. Anales de Psicología, 30(1), 211-220. http://dx.doi.org/10.6018/ analesps.30.1.135051

Mayseless, O. (1996). Attachment patterns and theiroutcomes. Human Development, 39, 206223. http://dx.doi.org/10.1 159/000278448 Medrano, A., Miranda, M., \& Figueras, V. M. (2017). Violencia de pareja contra las mujeres en México: Una mirada a la atención del sector salud desde una perspectiva interseccional. Multidisciplinary Journal of Gender Studies, 6, 1231-1262. http://dx.doi.org/10.17583/ generos.2017.2131

Melero, R., \& Cantero, M. J. (2008). Los estilos afectivos en la población española: Un cuestionario de evaluación del apego adulto. Clínica y Salud, 19(1), 83-98.

Mikulincer, M., \& Horesh, N. (1999). Adult attachment style and the perception of others: The role of projective mechanisms. Journal of Personality and Social Psychology, 76, 10221034. http://dx.doi.org/10.1037/00223514.76.6.1022

Moral, M. V., \& Ovejero, A. (2004). Jóvenes, globalización y postmodernidad: Crisis de la adolescencia social en una sociedad adolescente en crisis. Papeles del Psicólogo, 25(87), 72-79.

Moral, M. V., \& Sirvent, C. (2008). Dependencias sentimentales $\mathrm{a}$ afectivas: Etiología, clasificación y evaluación. Revista Española de Drogodependencia, 33(2), 145-167.

Moral, M. V., \& Sirvent, C. (2009). Dependencia afectiva y género: Perfil sintomático diferencial en dependientes afectivos españoles. Revista Interamericana de Psicología, 43(2), 230240.

Moral, M. V., García, A., Cuetos, G., \& Sirvent. C. (2017). Violencia en el noviazgo, dependencia emocional y autoestima en adolescentes y 
jóvenes españoles. Revista Iberoamericana de Psicología y Salud, 8(2), 96-107. https://doi. org/10.23923/i.rips.2017.08.009

Nóblega, M. S., Traverso, M. P., Ugarte, A. S., \& Caballero, M. G. (2017). Factores sociodemográficos explicativos del guión de base segura materno. Revista de Psicología, 35(2), pp. 575-604. http://dx.doi. org/10.18800/psico.201702.007.

Novo, M., Herbón, J., \& Amado, B. G. (2016). Géneroyvictimización: Efectos en la evaluación de la violencia psicológica sutil y manifiesta, apego adulto y tácticas de resolución de conflictos. Revista Iberoamericana de Psicología y Salud, 7(2), 89-97. https://doi. org/10.1016/i.rips.2016.05.002

Novo, M., Fariña, F., Seijo, D., \& Arce, R. (2012). Assessment of a community rehabilitation program in convicted male intimate-partner violence offenders. International Journal of Clinical and Health Psychology, 12, 219-234. Recuperado de http://www.aepc.es/ijchp/ articulos pdf/iichp-408.pdf

Penagos, A. Rodríguez, M., Carrillo, S., \& Castro, J. (2006) Apego, relaciones románticas y autoconcepto en adolescentes bogotanos. Universitas Psychologica, 5, 21-36.

Petani, R. (2011). Correlation between family interaction and adolescent's attitudes. The Andragogic Perspectives, 3, 10-22.

Petruccelli, F., Diotaiuti, P., Verrastro, V., Petrucelli, I., Federico, R., Martinetti, G., Fossati, A., Di Giannantonio, M., \& Janiri, L. (2014). Affective dependence and aggression: An exploratory study. BioMed Research International, 1-11. http://dx.doi.org/10.1155/2014/805469

Pradas, E., \& Perles, F. (2012). Resolución de conflictos de pareja en adolescentes, sexismo y dependencia emocional. Quaderns de Psicología, 14(1), 45-60.

Redondo, J., Inglés, C. J., \& García, K. (2017). Papel que juega la edad en el noviazgo de estudiantes de la Universidad Pontificia Bolivariana de Bucaramanga. Diversitas: perspectivas en Psicología, 13(1), 51 64. https://dx.doi.org/10.15332/s17949998.2017 .0001 .04

Redding, E. M., Ruiz, M. T., Fernández, J., \&
Guijarro, M. (2017). Gender inequality and violence against women in Spain, 2006-2014: Towards a civilizited society. Gaceta Sanitaria, 31, 82-88. https://dx.doi.org/10.1016/i. gaceta.2016.07.025

Rodríguez de Medina, I. (2013). La dependencia emocional en las relaciones interpersonales. Revista Electrónica de Investigación Docencia Creativa, 2, 143-148.

Sánchez, M. (2011). Apego en la infancia y Apego adulto: influencia en las relaciones amorosas y sexuales [Tesis de Maestría]. Salamanca: Universidad de Salamanca.

Sánchez, M. (2016). Los estilos de apego en mujeres con y sin violencia conyugal. Revista de Investigación en Psicología, 19(1), 35-49.

Sangrador, J. L. (1993). Consideraciones psicosociales sobre el amor romántico. Psicothema, 5(1), 181-196. Recuperado de http://www.psicothema.com/pdf/1 137.pdf

Santamaría, J. J., Merino, L., Montero, E., Cano, M., Fernández, T., Cubero, P., López, O., \& González-Bueso, V. (2015). Perfil psicopatológico de pacientes con Dependencia Emocional. Cuadernos de Medicina Psicosomática, 1 16, 36-46.

Simpson, W., \& Rholes, S. (1998). Attachment theory and close relationships. New York, NY: The Guildford Press.

Sirvent, C. (2004, Octubre). Adicción al amor y otras dependencias sentimentales. Encuentro de Profesionales en Drogodependencias y Adicciones, Chiclana, España.

Sirvent, C., \& Moral, M. V. (2005). Inventario de Relaciones Interpersonales y Dependencias Sentimentales IRIDS-100. Madrid, España: Servicio de Publicaciones de Fundación Instituto Spiral.

Sirvent, C., \& Moral, M. V. (2007). La dependencia sentimental. Anales de Psiquiatría, 23(3), 9394.

Siödin, A. K., Wallinius, M., Billstedt, E., Hofvander, B., \& Nilsson, T. (2017). Dating violence compared to other types of violence: Similar offenders but different victims. European Journal of Psychology Applied to Legal Context, 9(2), 83-91. https://doi. org/10.1016/i.ejpal.2017.03.001 
Smith-Ełxeberría, K., Ortiz-Barón, M. J., \& Apodaca-Urquijo, P. (2014). Experiencias e interacciones de la familia de origen y su influencia en las relaciones afectivas de los adultos jóvenes. Apuntes de Psicología, 32(2), 127-136.

Sophia, E., Tavares, H., \& Zilberman, M. (2007). Pathological love: Is it a new psychiatric disorder? Revista Brasileña de Psiquiatría, 29(1), 55-620. http://dx.doi.org/10.1590/ $\underline{51516-44462007000100016}$

Urbiola, I., \& Estévez, A. (2015). Dependencia emocional y esquemas desadaptativos tempranos en el noviazgo de adolescentes y ióvenes. Psicología Conductual, 23(3), 571 587.

Waters, E., \& Beauchine, T. (2003). Are there really patterns of attachment? Comment on Fraley and Spieker's. Developmental Psychology, 39, 417-422. http://dx.doi. org/10.1037/0012-1649.39.3.417

Yárnoz, S., Alonso-Arbiol, I., Plazaola, M., \& Sainz, L. M. (2001). Apego en adultos y percepción de los otros. Anales de Psicología, 17(2), 159-170. 